\title{
微纳分级结构碳酸钙中空微球的可控制备
}

\author{
邹俭鹏 ${ }^{1}$, 杨洪志 ${ }^{1}$, 肖 平 ${ }^{2}$, 潘一峰 ${ }^{2}$ \\ (1.中南大学 粉末冶金国家重点实验室，长沙 410083; 2. 中南大学 湘雅医院，长沙 410008)
}

摘 要: 以 $\mathrm{CaCl}_{2}$ 和 $\mathrm{Na}_{2} \mathrm{CO}_{3}$ 为反应原料, 以聚乙烯吡咯烷酮(PVP)和十二烷基磺酸钠( $\mathrm{SDSN}$ )为模板剂, 在 $50^{\circ} \mathrm{C}$ 采用 化学沉淀反应, 干燥、被烧后成功制备了具有微纳分级结构的 $\mathrm{CaCO}_{3}$ 中空微球。采用扫描电子显微镜、透射电子 显微镜和 $\mathrm{X}$ 射线衍射等检测手段对所制备的样品形貌、结构进行了表征，结果显示: 所制备的微纳分级结构 $\mathrm{CaCO}_{3}$ 中空微球直径为 4 6 $4 \mathrm{~m}$, 壳壁由直径约 $60 \mathrm{~nm}$ 的 $\mathrm{CaCO}_{3}$ 颗粒组成, 壳层厚度约为 $200 \mathrm{~nm}, \mathrm{CaCO}_{3}$ 中空微球晶相组 成为方解石和球霰石的共混体。同时, 在反应温度为 $50^{\circ} \mathrm{C} 、 \mathrm{PVP}$ 添加量为 $0.4 \mathrm{~g}, \mathrm{SDSN}$ 浓度为 $0.1 \mathrm{~mol} / \mathrm{L}$ 的条件下, 所制备的微纳分级结构 $\mathrm{CaCO}_{3}$ 中空微球分散性好，且形貌比较完整。

关 键 词: 微纳分级结构; 碳酸䥻中空微球; 作用机理; 模板法

中图分类号: R318 文献标识码: A

\section{Controllable Fabrication of Calcium Carbonate Hollow Microspheres with Micro-nano Hierarchical Structure}

\begin{abstract}
ZOU Jian-Peng ${ }^{1}$, YANG Hong-Zhi ${ }^{1}$, XIAO Ping ${ }^{2}$, PAN YI-Feng ${ }^{2}$
(1. State Laboratory of Powder Metallurgy, Central South University, Changsha 410083, China; 2. Xiangya Hospital, Central South University, Changsha 410008, China)

Abstract: With polyvinyl pyrrolidone (PVP) and sodium dodecyl sulfonate (SDSN) as the template, calcium carbonate hollow microspheres with micro-nano hierarchical structure were successfully synthesized using sodium carbonate and calcium chloride as starting materials through a precipitation reaction method at reaction temperature of $50^{\circ} \mathrm{C}$. The products were characterized by scanning electronic microscopy (SEM), transmission electron microscope (TEM), X-ray diffraction (XRD) and other detection methods. The results show that the hollow calcium carbonate microspheres with micro-nano hierarchical structure are about 4-6 $\mu \mathrm{m}$ in diameter. The shell thickness of calcium carbonate hollow microspheres is about $200 \mathrm{~nm}$, which consists of calcium carbonate particles with size about $60 \mathrm{~nm}$. The phase of calcium carbonate hollow microspheres is composed of calcite and vaterite. Excellent dispersibility and spherical morphology of calcium carbonate hollow microspheres can be achieved with addition of $0.1 \mathrm{~mol} / \mathrm{L} \mathrm{SDSN}$ and $0.4 \mathrm{~g}$ PVP consequently.
\end{abstract}

Key words: micro-nano hierarchical structure; calcium carbonate hollow microspheres; mechanism; template

微纳分级结构作为一种核壳结构, 是由纳米尺 度的基本结构单元相互堆叠在一起作为壳构成的微
米尺度的中空微球。微纳分级结构拥有优异的结构 特点(密度低、比表面积大、稳定性好、表面可渗透

收稿日期：2015-12-29; 收到修改稿日期：2016-03-24

基金项目：国家自然科学基金(51274248); 国际科技合作专项项目(2013DFA31440) National Natural Science Foundation of China (51274248); International S\&T Cooperation Program of China (2013DFA31440)

作者简介: 邹俭鹏(1976-), 男, 博士, 教授. E-mail: zoujp@csu.edu.cn

通讯作者：肖 平, 教授, 主任医师. E-mail: xiaoping.x@163.com 
性好), 一直是材料科学的前沿研究领域之一 ${ }^{[1]}$ 。 $\mathrm{CaCO}_{3}$ 作为一种用途广泛的化工产品, 因原料低廉 易得、生产工艺简单、性能稳定等特点, 已成为国 内外研究的热点, 使其在橡胶、塑料、造纸、涂料、 纺织、食品、牙膏等领域得到广泛应用 ${ }^{[2-10]}$ 。而具 有微纳分级结构的 $\mathrm{CaCO}_{3}$ 中空微球由于其独特的结 构、光学性能和表面性质, 使得 $\mathrm{CaCO}_{3}$ 的应用范围 进一步扩大 ${ }^{[11-13]}$, 在制药学、生物学及化妆品工业 等方面具有潜在的应用前景 ${ }^{[14-15]}$ 。

目前, 多孔 $\mathrm{CaCO}_{3}$ 微球的制备主要是利用溶液 合成方法, 借助球形模板剂及各种添加剂的调控作 用，制备出形貌和结构有效可控的碳酸钙粒子 ${ }^{[16-18]}$ 。 但添加剂与模板剂多为表面活性剂与高聚物, 在实 验中存在一些不足, 如表面活性剂对实验条件要求 比较苛刻, 使得材料制备工艺复杂 ${ }^{[19]}$; 模板剂与无 机材料壳层间作用力弱, 在包覆过程中需要对材料 表面进行改性 ${ }^{[20]}$ 。因此, 选择合适的模板剂是实现 碳酸钻简易制备及形貌可控的必要前提。

本研究采用一种简单的方法, 用生物相容性 较好的表面活性剂 PVP、SDSN 作为模板, 采用自 组装方式, 在 $50^{\circ} \mathrm{C}$ 反应条件并经干燥㷽烧后制备 出具有微纳分级结构的 $\mathrm{CaCO}_{3}$ 中空微球。此方法 对制备条件要求较低, 且制备简单、成本低, 实现 了 $\mathrm{CaCO}_{3}$ 形貌的可控化, 有望实现 $\mathrm{CaCO}_{3}$ 大规模 生产。

\section{1 实验方法}

\section{1 原料}

无水碳酸钠 $\left(\mathrm{NaCO}_{3}\right), \mathrm{AR}$, 西陇化工股份有限
公司; 无水氯化钙 $\left(\mathrm{CaCl}_{2}\right), \mathrm{AR}$, 西陇化工股份有限 公司; 无水乙醇, $\mathrm{AR}$, 天津市恒兴化学试剂制造有 限公司; 十二烷基磺酸钠(SDSN), CP, 西陇化工股 份有限公司; 聚乙烯吡咯烷酮(PVP), CP, 湖南汇虹 试剂有限公司; 蒸馏水为实验室自制。

\section{2 微纳分级结构 $\mathrm{CaCO}_{3}$ 中空微球的制备}

准确配制浓度为 $0.1 \mathrm{~mol} / \mathrm{L}$ 的 $\mathrm{CaCl}_{2}$ 溶液、 $\mathrm{Na}_{2} \mathrm{CO}_{3}$ 溶液和 SDSN 溶液, 在磁力搅拌条件下, 称 取 $0.4 \mathrm{~g}$ PVP 分别与 $100 \mathrm{~mL} \mathrm{CaCl}$ 溶液、 $200 \mathrm{~mL}$ $\mathrm{Na}_{2} \mathrm{CO}_{3}$ 溶液混合。然后在磁力搅拌的条件下将 $100 \mathrm{~mL}$ 的 $\mathrm{CaCl}_{2} / \mathrm{PVP}$ 混合溶液逐滴滴入到 $100 \mathrm{~mL} \mathrm{SDSN}$ 溶 液中, 并持续摚拌 $30 \mathrm{~min}$, 然后将混合液置于低温 恒温槽中并调节反应温度, 将 $200 \mathrm{~mL} \mathrm{Na} \mathrm{CO}_{3} / \mathrm{PVP}$ 溶液, 逐滴滴加到上述混合溶液中, 控制滴加速度, 使滴加时间在 $0.5 \sim 1 \mathrm{~h}$ 范围内, 反应流程如图 1 所示; 反应结束后, 再持续搅拌 $4 \mathrm{~h}$, 然后将反应产物静置 $12 \mathrm{~h}$ 过滤; 将沉淀用蒸馏水和无水乙醇分别洗涤 3 次, 然后将沉淀放入电热鼓风干燥箱中, 在 $80^{\circ} \mathrm{C}$ 的 条件下干燥 $12 \mathrm{~h}$, 然后放入马弗炉中 $350^{\circ} \mathrm{C}$ 炦烧 $4 \mathrm{~h}$, 收集样品备用。

\section{3 样品表征}

使用扫描电子显微镜(SEM，美国 FEI，Nova Nano SEM 230)观察 $\mathrm{CaCO}_{3}$ 的表观形貌和颗粒大小; 使用日本电子公司的透射电子显微镜(TEM，JEM$2100 \mathrm{~F}$ )观察样品的结构及形貌; 利用 DX-2000 X 射 线单色衍射仪(XRD)对样品的晶相组成进行测定。 测试条件为 $\mathrm{Cu} K \alpha(\lambda=0.15406 \mathrm{~nm})$, 管电压 $35 \mathrm{kV}$, 管电流 $25 \mathrm{~mA}$; 采用 $\mathrm{KBr}$ 压片技术在在 NICOLET500 红外光谱仪对 $\mathrm{CaCO}_{3}$ 粉末样品进行红外光谱 测定。

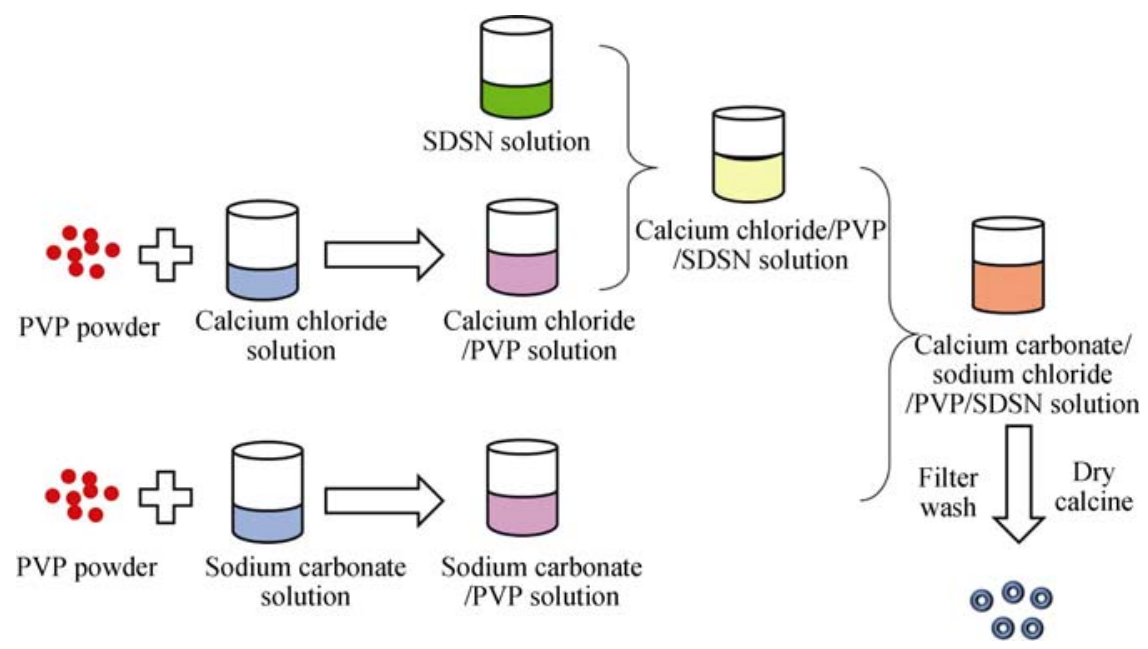

图 1 反应流程图

Fig. 1 The flow chart of reaction 


\section{2 结果与讨论}

\section{$2.1 \mathrm{CaCO}_{3}$ 中空微球的形貌}

图 2 为在反应温度为 $50^{\circ} \mathrm{C}, \mathrm{PVP}$ 添加量为 $0.4 \mathrm{~g}$, SDSN 浓度为 $0.1 \mathrm{~mol} / \mathrm{L}$ 的条件下制备的微纳分级结 构 $\mathrm{CaCO}_{3}$ 中空微球的 SEM 照片, 可以看到 $\mathrm{CaCO}_{3}$ 微球的表面形貌。从图 2(a)可以看出, 所制备的 $\mathrm{CaCO}_{3}$ 微球大小比较均一, 分散性较好, 直径在 4 6 $\mu \mathrm{m}$; 从破损的球形粒子(图 2(b))及放大图(图 2(c)) 可以看出, 所制备的微球具有中空核壳结构, 壳壁是由颗粒粒径约 $60 \mathrm{~nm}$ 的 $\mathrm{CaCO}_{3}$ 颗粒组成, 壳 的厚度约为 $200 \mathrm{~nm}$ 。

\section{$2.2 \mathrm{CaCO}_{3}$ 中空微球的微纳分级结构分析}

由 TEM 照片可以看出, 所制备的微纳分级结 构 $\mathrm{CaCO}_{3}$ 微球为中空结构(如图 3(a)所示), 组成壳 壁的 $\mathrm{CaCO}_{3}$ 粒径大小在 $58 \mathrm{~nm}$ 左右(如图 3(b))。通 过电子衍射图像(图 3(c))可以表明所制备的微纳分 级结构 $\mathrm{CaCO}_{3}$ 微球具有多晶结构, 由于通过沉淀化 学反应所制备的微纳分级结构 $\mathrm{CaCO}_{3}$ 微球粒径较大, 使得视野区域范围内晶粒较少, 从而使得多晶环不 是特别清晰。结合 XRD 分析, 这种多晶结构为球霰 石和方解石的共混体。通过 HRTEM 照片中晶面间 距的测量, 从而对晶面进行了标定(图 3(d))。其中,
(110)为方解石晶面, (102)、(006)为球霰石晶面。

\section{$2.3 \mathrm{CaCO}_{3}$ 中空微球的晶型结构}

图 4 为微纳分级结构 $\mathrm{CaCO}_{3}$ 中空微球的 XRD 图谱。由图 4 中可知, 所制备的 $\mathrm{CaCO}_{3}$ 中空微球为 球霰石与方解石两种晶型结构。其中, 方解石属于 菱方晶系, 与 PDF 卡片 83-1762 比较, 其出峰位置和 各峰相对强度比较一致, 在方解石型 $\mathrm{CaCO}_{3}$ 的主要 特征衍射峰位置 $\left(2 \theta=29.40^{\circ}, 35.90^{\circ}, 39.50^{\circ}, 43.1^{\circ}\right.$ 等 左右)均出峰; 但同时依据 PDF 卡片 72-0506, 在球霰 石的特征衍射峰位置 $\left(2 \theta=20.90^{\circ}, 24.82^{\circ}, 27.00^{\circ}\right.$, $32.65^{\circ}$ 等左右)也出现峰, 但峰强度较方解石峰弱, 表明样品中含有少量的球霰石晶型碳酸钙。

图 5 为不同添加剂下, 微纳分级结构 $\mathrm{CaCO}_{3}$ 中 空微球的 XRD 图谱。由 XRD 图可知，在仅有 SDSN 存在的情况下, 所制备的 $\mathrm{CaCO}_{3}$ 中空微球均为方解 石晶型，并未出现球霰石晶型，原因可能在于 PVP 有机质通过与 $\mathrm{Ca}^{2+}$ 在界面上静电匹配、几何匹配和 立体化学匹配等作用，控制着 $\mathrm{CaCO}_{3}$ 的成核与生长， 同时, PVP 的浓度影响着 PVP 分子在溶液中的聚集 状态，使其呈现线形、类圆形、网形等不同的立体 化学构象 ${ }^{[21]}$, 当 PVP 高分子链为类圆形时, 由于 $\mathrm{PVP}$ 中基团 $\mathrm{C}=\mathrm{O}$ 中的氧原子可以从各个方向吸引 溶液中的 $\mathrm{Ca}^{2+}$, 因而形成形貌为球形的球霰石 $\mathrm{CaCO}_{3}$ 晶体并使其稳定存在。
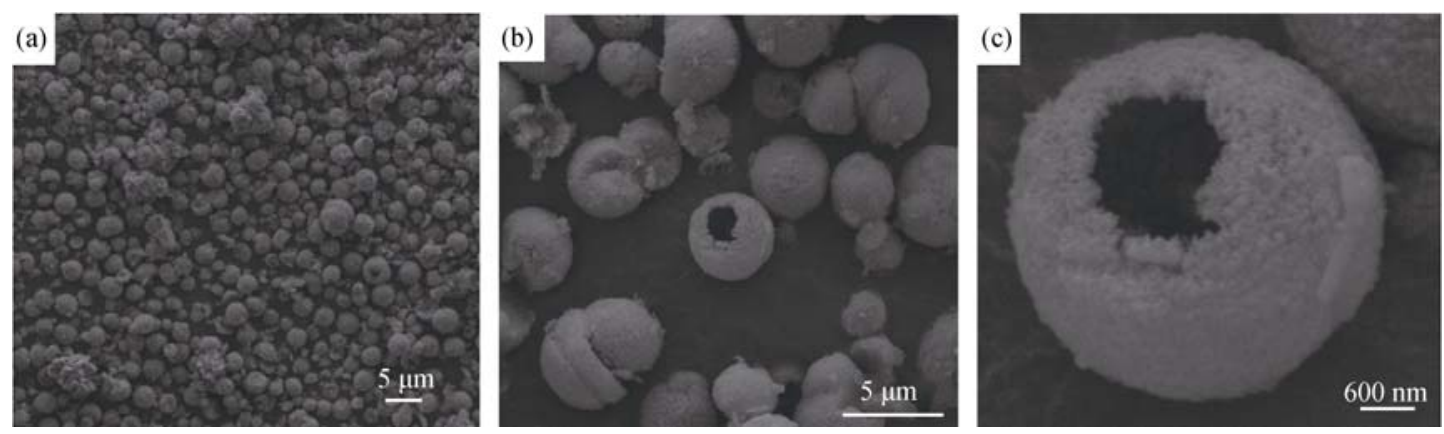

图 2 微纳分级结构 $\mathrm{CaCO}_{3}$ 中空微球的 SEM 照片

Fig. 2 SEM micrographs of calcium carbonate hollow microspheres with micro-nano hierarchical structure
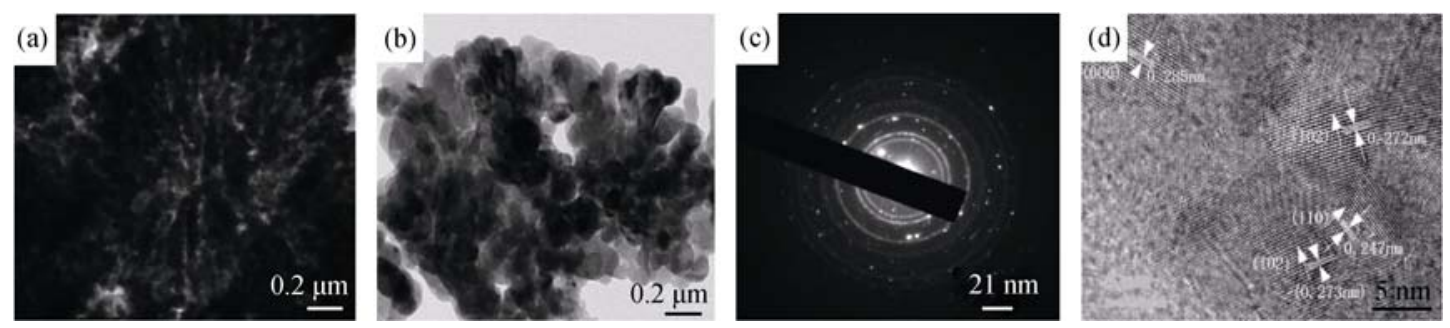

图 3 微纳分级结构 $\mathrm{CaCO}_{3}$ 中空微球的 TEM 照片

Fig. 3 TEM micrographs of calcium carbonate hollow microspheres with micro-nano hierarchical structure a: Morphology of calcium carbonate hollow microspheres; b: Morphology of nano-calcium carbonate on the micro-spherical shell wall; c: Electron diffraction pattern of calcium carbonate hollow microspheres; $d$ : Crystal calibration in HRTEM) 


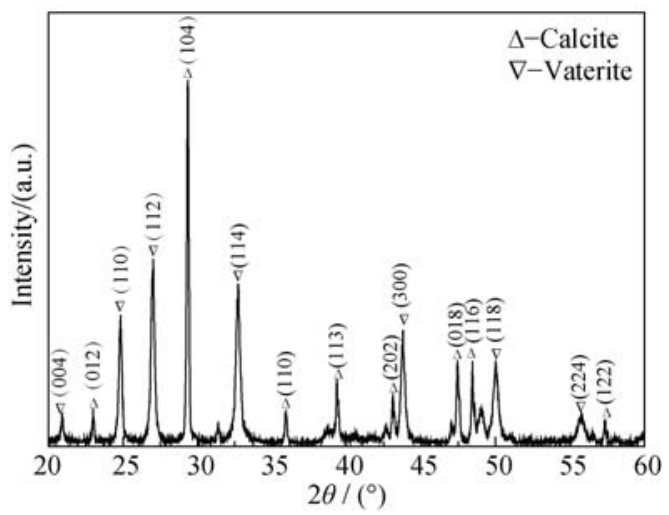

图 4 微纳分级结构 $\mathrm{CaCO}_{3}$ 中空微球 XRD 图谱

Fig. 4 XRD patterns of calcium carbonate hollow microspheres with micro-nano hierarchical structure

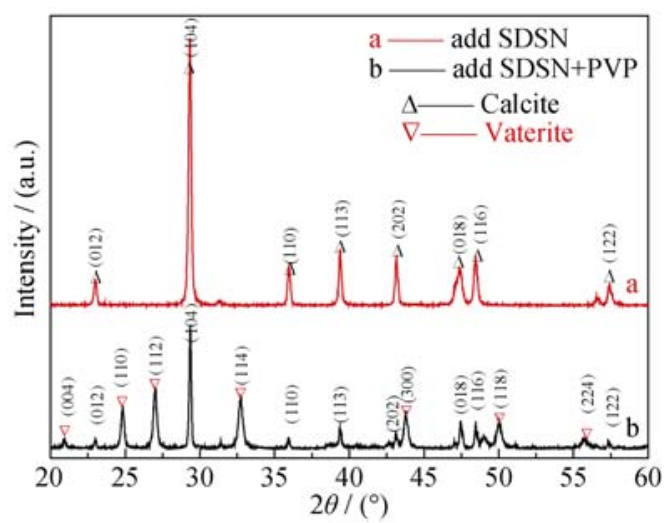

图 5 不同添加剂下, 微纳分级结构 $\mathrm{CaCO}_{3}$ 中空微球的 XRD 图谱

Fig. 5 XRD patterns of calcium carbonate hollow microspheres with micro-nano hierarchical structure at different additives

\section{4 微纳分级结构 $\mathrm{CaCO}_{3}$ 中空微球的 IR 分析}

图 6 为微纳分级结构 $\mathrm{CaCO}_{3}$ 中空微球的 IR 图。 $876 \mathrm{~cm}^{-1} 、 711 \mathrm{~cm}^{-1}$ 分别为方解石面外弯曲振动 $\left(\mathrm{v}_{2}\right) 、$ 剪式弯曲振动 $\left(\mathrm{v}_{4}\right)$ 的特征峰 ${ }^{[22]}$, 且 $\mathrm{v}_{2}$ 峰强而尖锐, 说明所制备的 $\mathrm{CaCO}_{3}$ 中空微球以方解石晶型为主; 同时, $1080 \mathrm{~cm}^{-1} 、 746 \mathrm{~cm}^{-1}$ 分别为球霰石对称伸缩振

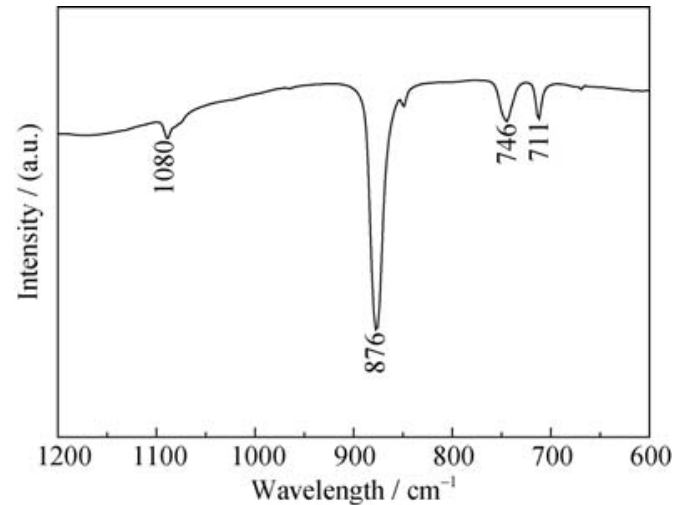

图 6 微纳分级结构 $\mathrm{CaCO}_{3}$ 中空微球的 IR 图谱

Fig. 6 IR spectrum of calcium carbonate hollow microspheres with micro-nano hierarchical structure

动 $\left(\mathrm{v}_{1}\right)$ 、剪式弯曲振动 $\left(\mathrm{v}_{4}\right)$ 的特征峰 ${ }^{[23]}$, 但球霰石晶 型 $\mathrm{CaCO}_{3}$ 的特征峰强度比较小, 说明有少量球霰石 晶型的存在。

\section{5 不同反应温度对微纳分级结构 $\mathrm{CaCO}_{3}$ 中 空微球形貌的影响}

为了研究温度对微纳分级结构 $\mathrm{CaCO}_{3}$ 中空微球 形貌的影响，实验中控制 $\mathrm{CaCl}_{2} 、 \mathrm{Na}_{2} \mathrm{CO}_{3} 、 \mathrm{SDSN}$ 的 浓度为 $0.1 \mathrm{~mol} / \mathrm{L}, \mathrm{PVP}$ 添加量为 $0.4 \mathrm{~g}$, 反应温度依 次为 $20^{\circ} \mathrm{C}, 40^{\circ} \mathrm{C}, 60^{\circ} \mathrm{C}, 80^{\circ} \mathrm{C}$ 时, 所制备的样品经 SEM 观察到的形貌如图 7 所示。在 $20^{\circ} \mathrm{C}$ 反应条件下 所制备的 $\mathrm{CaCO}_{3}$ 除球形实心结构外，还存在六面体 结构(图 7(a)); 随着温度的升高, 在 $40^{\circ} \mathrm{C}$ 反应条件 下制备的 $\mathrm{CaCO}_{3}$ 球形度提高, 从破损的微球可以看 出所制备的 $\mathrm{CaCO}_{3}$ 呈中空结构(图 7(b)); 但继续升 高反应温度，在 $60^{\circ} \mathrm{C}$ 时反应所制备的 $\mathrm{CaCO}_{3}$ 虽具有 中空结构, 但微球破损严重, 并出现异常长大的颗 粒(图 7(c)); 而在 $80^{\circ} \mathrm{C}$ 条件下反应制备的 $\mathrm{CaCO}_{3}$ 呈 米粒状, 不具有中空结构(图 7(d))。方解石是热力学 稳定相，在较低温度下，反应倾向于形成具有菱形 结构的方解石碳酸铝 ${ }^{[24]}$, 虽然 SDSN 球形类胶束簇 形成的核和由 PVP 长链包绕在类胶束簇的表面所形 成的“核一壳”式复合物 ${ }^{[25]}$, 在 $\mathrm{CaCO}_{3}$ 沉淀过程中起
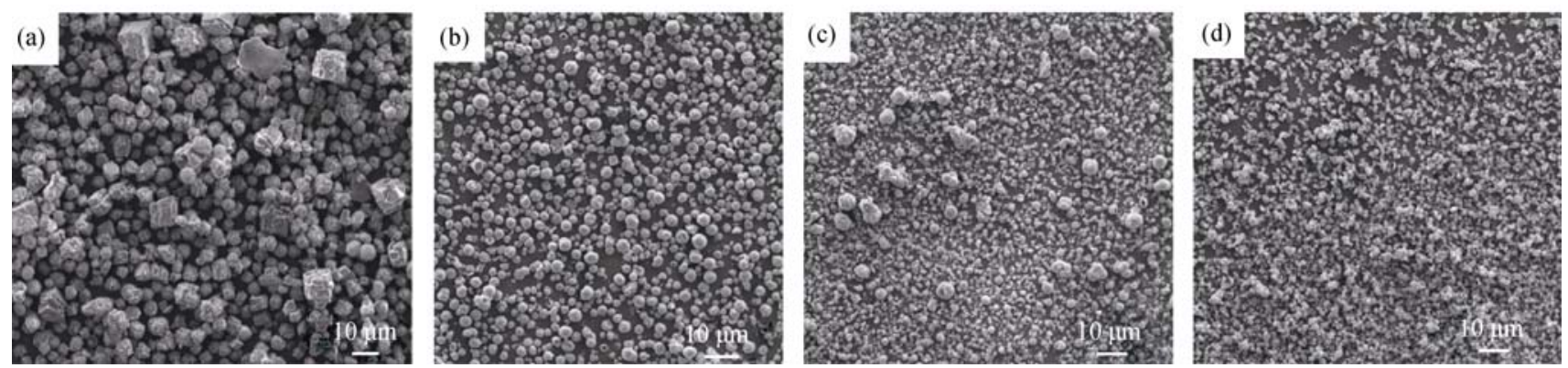

图 7 不同反应温度对微纳分级结构 $\mathrm{CaCO}_{3}$ 中空微球的影响

Fig. 7 Influence of the reaction temperature on calcium carbonate hollow microspheres with micro-nano hierarchical structure (a) $20^{\circ} \mathrm{C}$; (b) $40^{\circ} \mathrm{C}$; (c) $60^{\circ} \mathrm{C}$; (d) $80^{\circ} \mathrm{C}$ 
到了模板作用, 但在较低温度下, 离子的生长速率 及扩散速率较慢, 反应所生成的无定形碳酸钙 (ACC)来不及扩散就通过静电作用吸附在 PVP 长链 上, 使其形成实心结构; 随着温度的升高, 离子的 生长速率及扩散速率提高, SDNS、PVP 对 $\mathrm{CaCO}_{3}$ 调控作用加强; 但温度为 $80^{\circ} \mathrm{C}$ 时得到米粒状 $\mathrm{CaCO}_{3}$, 原因可能在于较高温度下, 晶体的配体关系发生改 变, 使其形成米粒状 $\mathrm{CaCO}_{3}{ }^{[26]}$ 。根据不同反应温度 下制得的样品 SEM 照片, 在 $40 \sim 60^{\circ} \mathrm{C}$ 范围可制得具 有微纳分级结构的 $\mathrm{CaCO}_{3}$ 中空微球, 对实验温度进 一步调节, 在 $50^{\circ} \mathrm{C}$ 条件下, 制得球形度及均一性较 好的具有微纳分级结构的 $\mathrm{CaCO}_{3}$ 中空微球。因此实 验的最佳反应温度定为 $50^{\circ} \mathrm{C}$ 。

\section{6 不同 SDSN 浓度对微纳分级结构 $\mathrm{CaCO}_{3}$ 中空微球形貌的影响}

为了研究不同浓度的 SDSN 溶液对微纳分级结 构 $\mathrm{CaCO}_{3}$ 中空微球形貌的影响, 实验中控制 $\mathrm{CaCl}_{2}$ 、 $\mathrm{Na}_{2} \mathrm{CO}_{3}$ 的浓度为 $0.1 \mathrm{~mol} / \mathrm{L}$, 且 PVP 添加量为 $0.4 \mathrm{~g}$, 控制 SDSN 的浓度依次为 $0.08 、 0.1 、 0.12 、 0.14 \mathrm{~mol} / \mathrm{L}$ 。 所制备的样品经 SEM 观察到的形貌如图 8 所示。实 验中, 所加入的 SDSN 浓度远大于其临界胶束浓度 $0.011 \mathrm{~mol} / \mathrm{L}^{[27]}$, 因此所加入的 SDSN 在溶液中以胶 束形式存在, 但在较低浓度 $0.08 \mathrm{~mol} / \mathrm{L}$ 时, 虽然 $\mathrm{SDSN}$ 的极性磺酸基 $\left(-\mathrm{HSO}_{3}{ }^{-}\right)$与 $\mathrm{Ca}^{2+}$ 间强烈静电作 用, 使 $\mathrm{Ca}^{2+}$ 在胶束的周围富集, 同时 $\mathrm{Ca}^{2+}$ 又会吸引 大量长链的 PVP 分子; 但 SDSN 胶束间相互簇化作 用较弱, 使这种由 SDSN 胶束形成的核和由 PVP 长 链包绕在类胶束表面所形成的 “核一壳”模板比较脆 弱, 在机械搅拌作用下容易破裂, 从图 8(a)中可以 看出有少量的中空微球存在, 但微球破损率很大; 增大 SDSN 溶液浓度, 胶束间簇化作用加剧, 形成 球形的胶束簇化束。同时大量的长链 PVP 分子相互 交织在球形胶束簇化束表面, 在 $\mathrm{CaCO}_{3}$ 生成过程中 起到模板作用, 同时还具有疏水分散的作用, 从图 8(b)可以看出, 所制备的 $\mathrm{CaCO}_{3}$ 微球大小比较均一, 分散性较好; 但继续增大 SDSN 浓度, 中空微球数
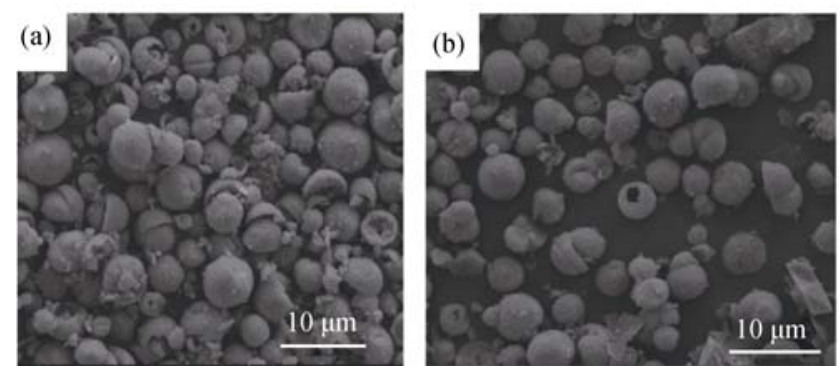

量减少, 出现实心微球, 原因可能是 SDSN 浓度增 大后，吸附在胶束簇表面的 $\mathrm{Ca}^{2+}$ 浓度增加，同时， $\mathrm{SDSN}$ 分子间、 $\mathrm{Ca}^{2+}$ 间的排斥力也增大，使球形胶束 簇化束失稳并随着反应的继续进行, 使后续生成的 $\mathrm{CaCO}_{3}$ 脱附占据中心区域, 从而形成实心结构。

\section{7 不同 PVP 添加量对微纳分级结构 $\mathrm{CaCO}_{3}$ 中空微球形貌的影响}

为了研究不同质量的 PVP 对微纳分级结构 $\mathrm{CaCO}_{3}$ 中空微球形貌的影响, 实验中控制 $\mathrm{CaCl}_{2}$ 、 $\mathrm{Na}_{2} \mathrm{CO}_{3} 、 \mathrm{SDSN}$ 的浓度为 $0.1 \mathrm{~mol} / \mathrm{L}$, 控制 PVP 的添 加量依次为 $0.32 、 0.4 、 0.8 、 1.2 \mathrm{~g}$, 所制备的样品 经 SEM 观察到的形貌如图 9 所示。当 PVP 添加量 较低时, 由图 9(a)可以看出, 生成的微球表面比较 粗粘, PVP 分子彼此间相互缠绕交织作用较弱; 当 PVP 添加量为 $0.4 \mathrm{~g}$ 时, 生成的微球表面比较光滑, 从照片来看, 二者均产生中空结构; 但继续增大 PVP 添加量, 反应生成了实心结构和空心破损中空 微球。PVP 浓度增大, 长链 PVP 分子缠绕交织加剧, SDSN 球形胶束簇的表面缠绕的聚合物链增多, 排 斥力增大, 同时引起 $\mathrm{CaCO}_{3}$ 粒子成核密度增大, 使 反应生成的沉淀颗粒增多，颗粒间团聚造成重量增 加，使后续生成的 $\mathrm{CaCO}_{3}$ 脱附占据中心区域; 同时 部分缠绕交织的长链 PVP 分子也起到模板作用, 但 其作用较弱, 在机械搅拌及后续热处理过程中, PVP 被去除, 得到如图 9(d)所示虽具有中空结构但 破损严重的 $\mathrm{CaCO}_{3}$ 微球。

\section{8 微纳分级结构 $\mathrm{CaCO}_{3}$ 中空微球的形成机 理探讨}

\subsection{1 反应的热力学分析}

实验中将 $\mathrm{Na}_{2} \mathrm{CO}_{3} 、 \mathrm{CaCl}_{2}$ 溶液混合, 反应过程 中方程式为:

$$
\mathrm{Na}_{2} \mathrm{CO}_{3}+\mathrm{CaCl}_{2}=2 \mathrm{NaCl}+\mathrm{CaCO}_{3} \downarrow
$$

同时, 各反应物标准热力学数据如表 1 所示。根据 热力学定律, 化学反应的标准摩尔焓变、标准摩尔 熵变随温度的变化很小, 可以忽略不计。所以式(1) 所示反应焓由式(2)、式(3)所示公式进行计算, 反应
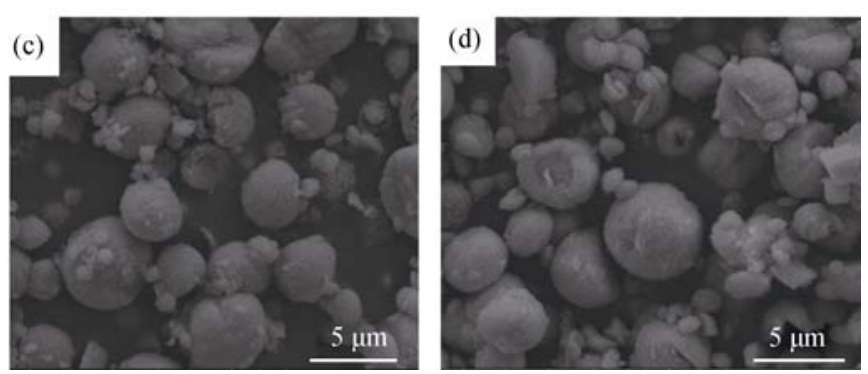

图 8 不同浓度 $\mathrm{SDSN}$ 对微纳分级结构 $\mathrm{CaCO}_{3}$ 中空微球的影响

Fig. 8 Influence of the concentration of SDSN on calcium carbonate hollow microspheres with micro-nano hierarchical structure (a) $0.08 \mathrm{~mol} / \mathrm{L}$; (b) $0.1 \mathrm{~mol} / \mathrm{L}$; (c) $0.12 \mathrm{~mol} / \mathrm{L}$; (d) $0.14 \mathrm{~mol} / \mathrm{L}$ 

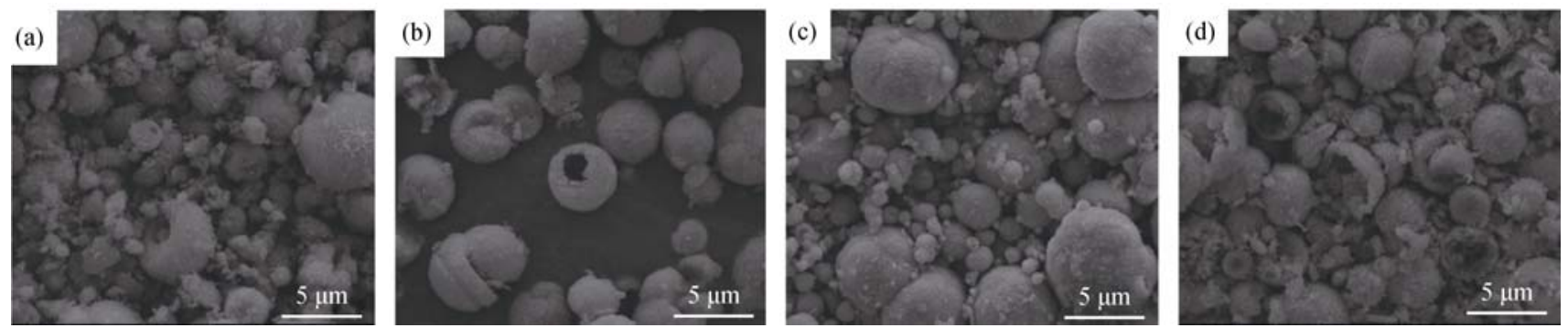

图 9 不同 PVP 添加量对微纳分级结构 $\mathrm{CaCO}_{3}$ 中空微球的影响

Fig. 9 Influence of the additive of PVP on calcium carbonate hollow microspheres with micro-nano hierarchical structure (a) $0.32 \mathrm{~g}$; (b) $0.4 \mathrm{~g}$; (c) $0.8 \mathrm{~g}$; (d) $1.2 \mathrm{~g}$

表 1 相关化合物的标准热力学数据

Table 1 Standard thermodynamic data of related compounds

\begin{tabular}{cccc}
\hline & $\begin{array}{c}\Delta_{\mathrm{f}} H_{\mathrm{m}}^{\ominus} / \\
\left(\mathrm{kJ} \cdot \mathrm{mol}^{-1}\right)\end{array}$ & $\begin{array}{c}\Delta_{\mathrm{f}} G_{\mathrm{m}}^{\ominus} / \\
\left(\mathrm{kJ} \cdot \mathrm{mol}^{-1}\right)\end{array}$ & $\begin{array}{c}S_{\mathrm{m}}^{\ominus} / \\
\left(\mathrm{J} \cdot \mathrm{mol}^{-1}\right)\end{array}$ \\
\hline $\mathrm{Na}_{2} \mathrm{CO}_{3}$ & -1130.7 & -1044.4 & 135.0 \\
$\mathrm{CaCl}_{2}$ & -795.4 & -748.8 & 108.4 \\
$\mathrm{NaCl}$ & -411.2 & -384.1 & 72.1 \\
$\mathrm{CaCO}_{3}$ & -1207.6 & -1129.1 & 91.7 \\
$($ calcite $)$ & & -1128.2 & 88.0 \\
$\begin{array}{c}\mathrm{CaCO} \\
(\text { vaterite })\end{array}$ & -1207.8 & & \\
\hline
\end{tabular}

的 Gibbs 自由能变化由式(4)或式(2)、(4)、(5)所示 公式计算。

$$
\begin{gathered}
\Delta \mathrm{r} H_{m}^{\ominus}=\sum v_{\mathrm{b}} \Delta_{\mathrm{f}} H_{\mathrm{m}}^{\ominus} \\
\Delta \mathrm{r} H_{m}(T) \approx \Delta_{\mathrm{r}} H_{\mathrm{m}}^{\ominus} \\
\Delta \mathrm{r} G_{m}(T) \approx \Delta_{\mathrm{r}} H_{\mathrm{m}}(T)-T \Delta_{\mathrm{r}} S_{\mathrm{m}}(T) \approx \Delta_{\mathrm{r}} H_{\mathrm{m}}^{\ominus}-T \Delta_{\mathrm{r}} S_{\mathrm{m}}^{\ominus} \\
\Delta_{\mathrm{r}} S_{\mathrm{m}}^{\ominus} \approx \sum v_{\mathrm{b}} S_{\mathrm{m}}^{\ominus}
\end{gathered}
$$

在 $50{ }^{\circ} \mathrm{C}$ 的反应条件下, 由公式及表中所列 数据, 计算得到反应摩尔焓变 $\Delta H_{\mathrm{m}}=-103.9 \mathrm{~kJ} / \mathrm{mol}$; $\Delta G_{\mathrm{m}}=-100.28 \mathrm{~kJ} / \mathrm{mol}, \Delta G_{\mathrm{m}}<0$, 反应可以自发发生; 并且, 式(1)反应属于复分解反应, 反应的离子方程 式为

$$
\mathrm{CO}_{3}{ }^{2-}+\mathrm{Ca}^{2+}=\mathrm{CaCO}_{3} \downarrow
$$

对于复分解反应，产物中生成沉淀或生成水或 生成挥发性气体, 反应就可以发生, 本实验所生成 的 $\mathrm{CaCO}_{3}$ 为沉淀，因此反应可以进行。

\subsection{2 反应过程机理分析}

图 10 为反应过程机理分析示意图。PVP 在水溶 液中以长链、类圆形、网形等形式存在, 实验中 PVP 与 $\mathrm{CaCl}_{2}$ 溶液混合时, 因其特殊的单体结构, 使其 作为螯合剂与 $\mathrm{Ca}^{2+}$ 结合在一起; 实验中 SDSN 的浓 度为 $0.1 \mathrm{~mol} / \mathrm{L}$, 远高于 $\mathrm{SDSN}$ 在水溶液中的临界胶 束浓度, 因此 SDSN 在溶液中以胶束形式存在, 并
通过簇化作用，胶束间形成球形的胶束簇化束。 SDSN 的极性磺酸基 $\left(-\mathrm{HSO}_{3}{ }^{-}\right)$与 $\mathrm{Ca}^{2+}$ 间的强烈静电 作用，使 $\mathrm{Ca}^{2+}$ 在簇化胶束的周围富集，并且降低晶 体的成核能，使其成为成核的活性点 ${ }^{[28]}$; 同时 $\mathrm{Ca}^{2+}$ 又会吸引大量长链的 PVP 分子, 使 PVP 分子包绕在 球形 SDSN 类胶束的簇的表面并与其部分发生疏水 作用, 球形胶束簇被 PVP 分子包围或被嵌入, 形成 了由 SDSN 球形类胶束簇形成的核和由 PVP 长链包 绕在类胶束簇的表面所形成的“核-壳”式复合物, 在 $\mathrm{CaCO}_{3}$ 沉淀过程中起到了模板作用，同时 PVP 长 链分子的位阻效应又在碳酸钙生成过程中起到了稳 定分散的作用; 当 $\mathrm{Na}_{2} \mathrm{CO}_{3}$ 溶液滴加到 $\mathrm{CaCl}_{2} / \mathrm{SDSN} /$ $\mathrm{PVP}$ 的混合溶液中时, $\mathrm{CO}_{3}{ }^{2-}$ 与富集在簇化束周围的 $\mathrm{Ca}^{2+}$ 结合生成非晶态的无定形 $\mathrm{CaCO}_{3}(\mathrm{ACC}), \mathrm{HSO}_{3}{ }^{-}$ 平行于 $\mathrm{CO}_{3}{ }^{2-}$ 所在的平面, 通过立体化学互补作用 可诱导方解石沿(001)面成核 ${ }^{[29]}$, 促进方解石晶体 的形成, 同时 PVP 分子的桥连作用使分散的 $\mathrm{CaCO}_{3}$ 颗粒彼此靠近, 为有序组装提供了可能, 使球形结 构更加圆整 ${ }^{[30]}$ 。既促进了 $\mathrm{CaCO}_{3}$ 的原位生成, 同时 长链分子间空间位阻效应又抑制了 $\mathrm{CaCO}_{3}$ 的生长, 在 SDSN 与 PVP 协同作用下，得到分散性良好且尺 寸在 4 6 $4 \mathrm{~m}$ 的方解石晶型碳酸钙; 同时, 类圆形的 PVP 立体化学构象能量较高, 基团 $\mathrm{C}=\mathrm{O}$ 中的氧原子 可以从各个方向吸引溶液中的 $\mathrm{Ca}^{2+}$, 进而形成形貌 为球形的球霰石 $\mathrm{CaCO}_{3}$ 晶体。然后产物经过滤、洗 涤、干燥、煅烧后, SDSN 与 PVP 被彻底除去, 形成 中空的具有微纳分级结构的 $\mathrm{CaCO}_{3}$ 微球。

\section{3 结论}

1) 通过 $\mathrm{CaCl}_{2} 、 \mathrm{Na}_{2} \mathrm{CO}_{3}$ 间的化学沉淀反应, 以 $\mathrm{SDSN} 、 \mathrm{PVP}$ 为表面活性剂, 在 $50^{\circ} \mathrm{C}$ 反应温度并经 干燥敾烧的条件下制备了具有微纳分级结构的 


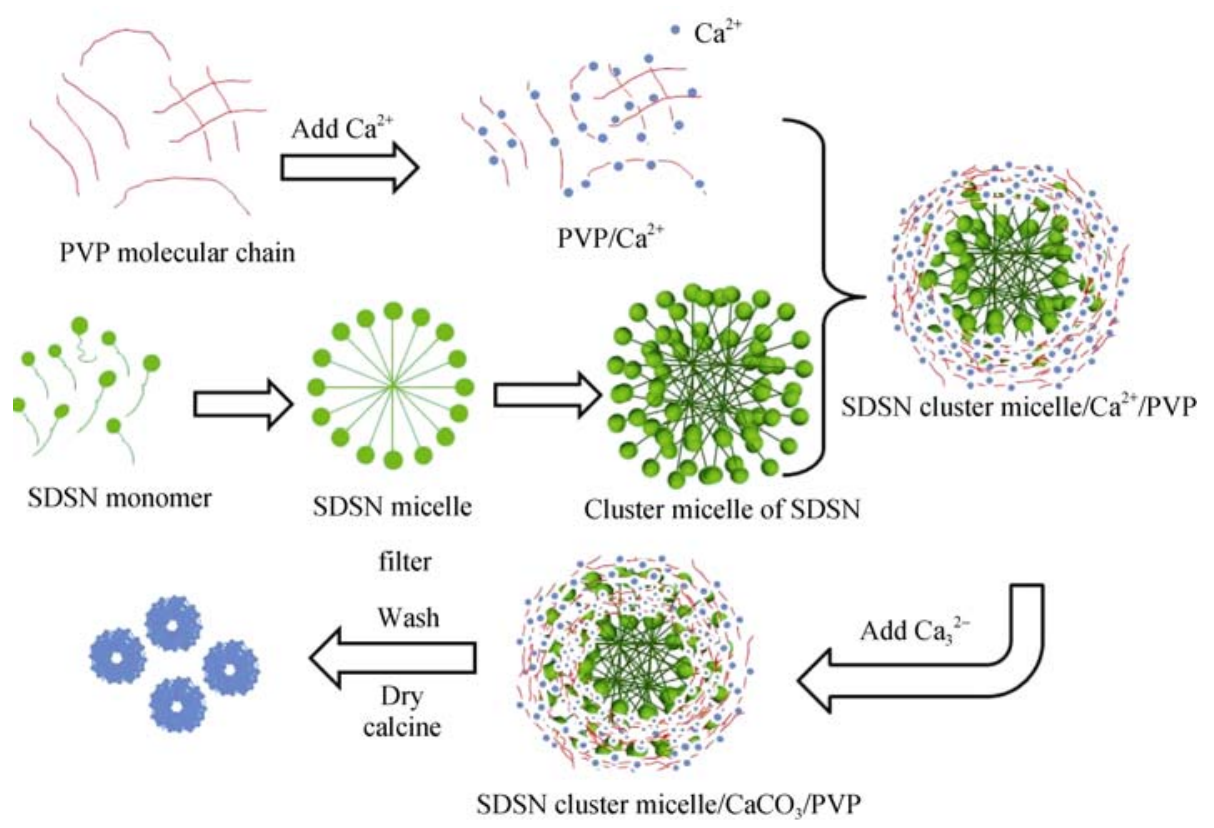

图 $10 \mathrm{SDSN}$ 与 PVP 调控生成 $\mathrm{CaCO}_{3}$ 中孔微球机理图

Fig. 10 The mechanism diagram of being modulated by SDSN and PVP for preparation of calcium carbonate hollow microspheres with micro-nano hierarchical structure

$\mathrm{CaCO}_{3}$ 中空微球。当 $\mathrm{SDSN}$ 浓度 $0.1 \mathrm{~mol} / \mathrm{L} 、 \mathrm{PVP}$ 添 加量为 $0.4 \mathrm{~g}$ 时, 所制备的碳酸钻中空微球大小均一, 分散性好。

2) PVP 在水溶液中的立体化学构象影响了球霰 石晶型碳酸钙晶体的成核与生长, 并进而影响了碳 酸钙的形貌; 有助于亚稳态球霰石晶型碳酸钻的稳 定，从而获得共混的方解石和球霰石碳酸钻。

3) SDSN 与 PVP 分子间的协同作用, 实现了对 碳酸钻形貌的可控性。

\section{参考文献:}

[1] LOU X W D, ARCHER L A, YANG Z. Hollow micro/ nanostructure: synthesis and applications. Advanced Materials, 2008, 20(21): 3987-4019.

[2] QI L, COLFEN H, ANTONIETTI M. Crystal design of barium sulfate using double-hydrophilic block copolymers. Angewandte Chemie International Edition, 2000, 39(3): 604-607.

[3] KRYSZTAFKIEWICZ A, JESIONOWSKI T, BINKOWSKI S. Precipitated silicas modified with 3-aminopropyltriethoxy silane. Colloids and Surfaces A: Physicochemical and Engineering Aspects, 2000, 173(1/2/3): 73-84.

[4] NAKA K, CHUJO Y. Control of crystal nucleation and growth of calcium carbonate by synthetic substrates. Chemistry of Materials, 2001, 13(10): 3245-3259.

[5] KATO T. SUGAWARA A, HOSODA N. Calcium carbonate- organic hybrid materials. Advanced Materials, 2002, 14(12):
869-877.

[6] DONG L H, CHU Y, LIU Y, et al. Surfactant-assisted fabrication $\mathrm{PbS}$ nanorods, nanobelts, nanovelvet-flowers and dendritic nanostructures at lower temperature in aqueous solution. Colloid and Interface Science, 2006, 301(2): 503-510.

[7] LI S Z, ZHANG H, WU J B, et al. Shape-control fabrication and characterization of the sirplane-like $\mathrm{FeO}(\mathrm{OH})$ and $\mathrm{Fe}_{2} \mathrm{O}_{3}$ nanostructures. Crystal Growth\&Design, 2006, 6(2): 351-353.

[8] GONG J Y, YU S H, QIAN H S, et al. Acetic acid-assisted solution process for growth of complex copper sulfide micrombes constructed by hexagonal nanoflakes. Chem. Mater., 2006, 18(8): 2012-2015.

[9] ZHENG D S, SUN S X, FAN W L, et al. One-step preparation of single-crystalline $\beta-\mathrm{MnO}_{2}$ nanotubes. Phys. Chem. B, 2005, 109(34): 16439-16443 .

[10] ZHANG M, WANG Z H, MO M S, et al. A simple approach to synthesize $\mathrm{KNiF}_{3}$ hollow spheres by solvothermal method. Materials Chemistry and Physics, 2005, 89(2/3): 373-378.

[11] SHEN Q, WEI H, ZHAO Y, et al. Morphological control of calcium carbonate crystals by polyvinylpyrrolidone and sodium dodecylbenzene sulfonate. Colloids and Surfaces A: Physicochemical and Engineering Aspects, 2004, 251(1/2/3): 87-91.

[12] WEN Y., XIANG L, JIN Y. Tribological investigation of PTFE composite filled with lead and rare earths-modified glass fiber. Materials Letters, 2003, 57 (16/17): 2553-2557.

[13] QI LM, LI J, MA J M. Biomimetic morphogenesis of calcium carbonate in mixed solutions of surfactants and double-hydrophilic block copolymers. Advanced Materials, 2002, 14(4): 300-303. 
[14] PENG C Y, ZHAO Q H, GAO C Y. Sustained delivery of doxorubicin by porous $\mathrm{CaCO}_{3}$ and chitosan/alginate multilayers- coated $\mathrm{CaCO}_{3}$ microparticles. Colloids and Surface A: Physicochemical and Engineering Aspects, 2010, 353(2/3): 132-139.

[15] WON Y H, JANG H S, CHUNG D W, et al. Multifunctional calcium carbonate microparticles: synthesis and biological applications. Journal of Materials Chemistry, 2010, 20(36): 7728-7733.

[16] HAN GUO-HUA, LI MEI-QIN, ZHU CHENG-YUN. Research progress of drug-loaded hollow microspheres. Journal of Shenyang Pharmaceutical University, 2011, 28(7): 575-580.

[17] ZHAO I N, WANG J K. Biomimetic synthesis of hollow microspheres of calcium carbonate crystals in the presence of polymer and surfactant. Colloids and Surfaces A: Physicochemical and Engineering Aspects, 2012, 393(5): 139-143.

[18] YUE I H, ZHENG Y F, JIN D I. Spherical porous framework of calcium carbonate prepared in the presence of precursor PS-PAA as template. Microporous and Mesoporous Materials, 2008, 113(1/2/3): 538-541.

[19] LI LIANG, ZHU YING-JIE, CAO SHAO-WEN, et al. Preparation and drug release properties of nanostructured $\mathrm{CaCO}_{3}$ porous hollow microspheres. Journal of Inorganic Materials, 2009, 24(1): $166-170$.

[20] WANG XIN, CHEN RI-XIN, YANG HUI. Study on wet surface modification of nanometer calcium carbonate. Non-metallic Mines, 2005, 28(6): 8-10.

[21] NIE QIU-LIN, ZHEN YI-FEN, YUE LIN-HAI, et al. Template induced synthesis of spherical $\mathrm{CaCO}_{3}$ by polyvinylpyrrolidone. Chinese Journal Of Inorganic Chemistry, 2003, 19(4): 445-448.

[22] TANG YANG-JUN, LI YOU-MING, SONG JING, et al. Structural characterization and thermal decomposition behavior of microsized and nanosized $\mathrm{CaCO}_{3}$. Acta Physico-Chimica Sinica, 2007, 23(5): $717-722$.

[23] WANG Y, MOO YX, CHEN C, et al. Fast precipitation of uniform $\mathrm{CaCO}_{3}$ nanospheres and their transformation to hollow hydroxyapatite nanospheres. J. Colloid Interface Science, 2010, 352(2): 393-400.

[24] ZENG LENG, HE QUAN-GUO, WU ZHAO-HUI. Preparation, surface modification and application of nano-calcium carbonate. Fine Chemical Intermediates, 2009, 39(4): 1-6.

[25] ZHAO LINA, KONG ZHI-GUO, WANG JI-KU. Preparation and mechanism of $\mathrm{CaCO}_{3}$ hollow microspheres. CIESC Journal, 2012, 63(6): 1976-1980.

[26] YU J G, GUO H, DAVIS S A, et al. Fabrication of hollow inorganic microspheres by chemically induced self-transformation. Advanced Functional Materials, 2006, 16(15): 2035-2041.

[27] HE GUO-XU, ZHANG LI, LI GUANG. Influence of inorganic salt on critical micelle concentration of sodium dodecyl sulfonate solution determined by conductivity method. Chemical Research, 2011, 22(2): 68-71.

[28] YANG XIAO-DENG, SHEN QIANG, XU GUI-YING. Crystallization of calcium carbonate using water-soluble macromolecules. Acta Physico-Chimica Sinica, 2010, 26(8): 2087-2095.

[29] ZHAO DONG-NAN, ZUO YI-SHI, MEI QIN, et al. Formation process and thermodynamic properties of calcite. Chinese Journal of Chemistry, 2007, 25(5): 592-595.

[30] YANU HUI, LI HUAN. The preparation of porous superfine calcium carbonate microspheres. Journal of Shanxi University of Science\&Technology, 2013, 31(5): 111-115. 